\title{
A CLOSED-FORM MODEL FOR THE SUPPORT STIFFNESS OF SPATIAL FLEXURE STRIPS WITH LIMITED TWIST
}

\author{
M. Nijenhuis, D.M. Brouwer \\ Mechanical Automation and Mechatronics, University of Twente, Enschede, The Netherlands \\ m.nijenhuis@utwente.nl, d.m.brouwer@utwente.nl
}

\begin{abstract}
The stiffness characteristics of flexure strips are important in the design and analysis of mechanisms that they constitute. This paper presents a closed-form parametric nonlinear model that describes the spatial error motions of a fixed-free flexure strip as a function of an arbitrary 3-D endload.
\end{abstract}

\section{Method}

Euler-Bernoulli beam theory with quadratic strain and curvature measures, and linear material behavior serve as a basis.

We have observed that many flexure mechanisms in practice contain flexure strips that experience only limited torsion deformation. Leveraging this, the general deformation is distinguished into

1. low-stiffness large-deflection motion, occurring in the principal deformation plane (PDP) of Fig. 1.

2. high-stiffness small-deflection motion, perpendicular to the PDP.

By assuming that only the motion in the PDP contributes to load-equilibrium, the deformed configuration admits an explicit solution as a function of the degrees of freedom and the applied loads. Closed-form expressions for the spatial error motions are then obtained as small disturbances with respect to the main deformed shape in the PDP.

For a practical formulation, the equations are formatted in terms of

- degrees of freedom (DOF) $\bar{u}_{y}, \bar{\varphi}_{z}, \bar{\varphi}_{x}$,

- applied loads $\bar{F}_{x}, \bar{F}_{z}, \bar{M}_{y}$, and

- error motions $\bar{u}_{x}, \bar{u}_{z}, \bar{\varphi}_{y}$.

\section{Results}

The expressions for the three error motions have the same structure, consisting of

- common linear elastic terms,

- nonlinear "kinematic" terms, quadratic in the DOF, and

- nonlinear "elastokinematic" terms, linear in the applied loads and quadratic in the DOF.

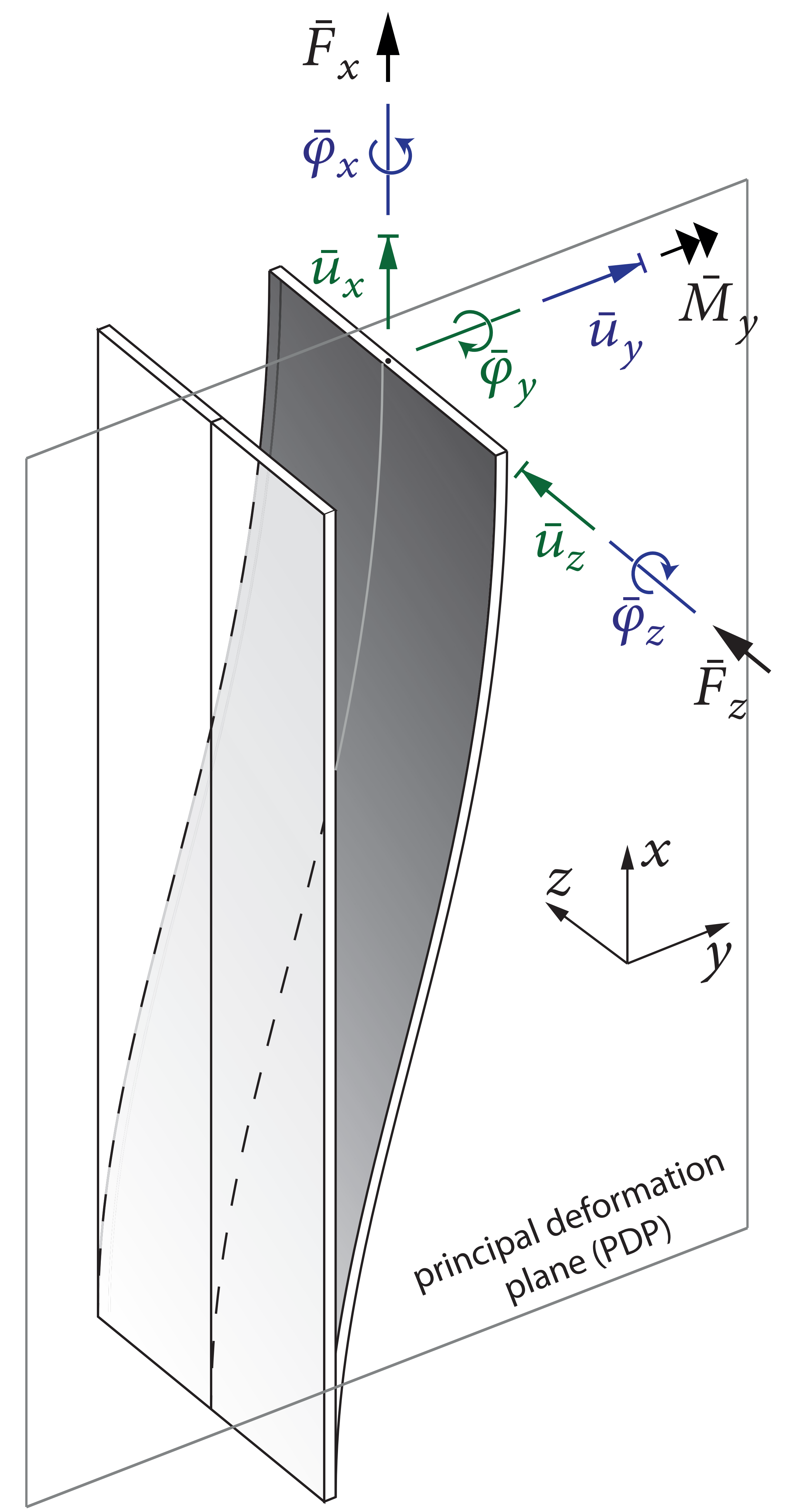

Figure 1: A fixed-free flexure strip with error motions (green), degrees of freedom (blue) and applied loads (black).

These parametric closed-form expressions capture the effects of distributed compliance in flexure strips.

\section{Validation}

The error motion expressions have been numerically validated for width-thickness ratios as small as 2 , and length-width ratios up to 10 , at various load levels, with 95\% accuracy.

\section{Conclusion}

By considering small spatial deformation with respect to a nominally 2-D deformed shape, accurate expressions are obtained for the geometrically nonlinear aspect of the 3-D behavior of a flexure strip. In practice, this models the performance-limiting reduction of support stiffness that accompanies movement in the intended degrees of freedom of a flexure strip. 